\title{
Heterogeneous nanozymatic activity of Hf oxo-clusters embedded in a metal-organic framework towards peptide bond hydrolysis
}

\author{
Jens Moons ${ }^{1}$, Alexandra Loosen ${ }^{1}$, Charlotte Simms ${ }^{1}$, Francisco de Azambuja ${ }^{1}$, Tatjana N. Parac-Vogt*1 \\ ${ }^{1}$ Department of Chemistry, KU Leuven, Celestijnenlaan 200F, 3001 Leuven, Belgium \\ *tatjana.vogt@kuleuven.be
}

\section{Table of Contents}

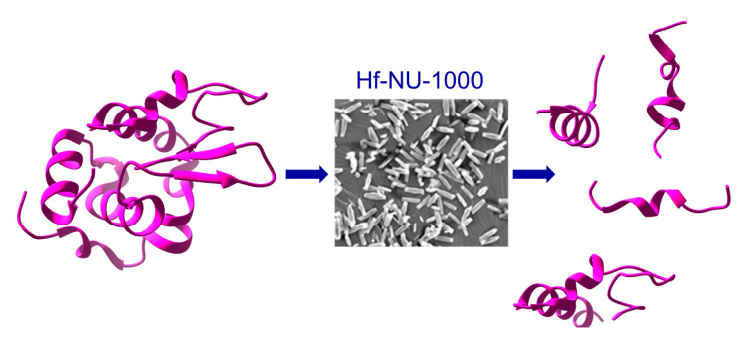

Hf-based NU-1000 metal organic framework as a hydrolytic nanozyme toward peptide bonds in dipeptides and hen egg white lysozyme protein revealed its greater stability and better recyclability than previous $\mathrm{Zr}$ / Hf-based nanozymes.

\begin{abstract}
The proteolytic activity of materials with enzyme-like activities is emerging as a robust, and effective alternative to natural enzymes. Herein, $\mathrm{Hf}_{6} \mathrm{O}_{8}$-based NU-1000 metal organic framework (Hf-MOF) is shown to act as a heterogeneous catalyst for the hydrolysis of peptide bonds under mild conditions. In the presence of Hf-MOF, glycylglycine model dipeptide was hydrolysed with a rate constant of $\mathrm{k}_{\mathrm{obs}}=8.33 \mathrm{x}$ $10^{-7} \mathrm{~s}^{-1}$ (half-life $\left(\mathrm{t}_{1 / 2}\right)$ of $231 \mathrm{~h}$ ) at $60{ }^{\circ} \mathrm{C}$ and $\mathrm{pD} 7.4$, which is significantly faster than the uncatalyzed reaction. Other Gly-X (X = Ser, Asp, Ile, Ala, and His) were also smoothly hydrolysed under the same conditions with similar rates, except for the faster reactions observed for Gly-His and Gly-Ser. Moreover, the $\mathrm{Hf}_{6} \mathrm{O}_{8}$-based NU-1000 MOF also exhibits a high selectivity in the cleavage of a protein substrate, hen egg white lysozyme (HEWL). Our results suggest that embedding $\mathrm{Hf}_{6} \mathrm{O}_{8}$ oxo-clusters is an efficient strategy to conserve the hydrolytic activity while smoothing the strong substrate adsorption previously observed for a discrete Hf oxo-cluster that hindered further development of their proteolytic potential. Furthermore, comparison with isostructural Zr-NU-1000 shows that although Hf variant afforded same
\end{abstract}


cleavage pattern towards HEWL but slightly slower reaction rates, it exhibited a larger stability window and better recyclability profile. Results suggest that these differences entail from intrinsic differences between $\mathrm{Hf}^{\mathrm{IV}}$ and $\mathrm{Zr}^{\mathrm{IV}}$ centers, and from the lower surface area of Hf-NU-1000 in comparison to Zr-NU1000.

\section{Introduction}

Metal-organic frameworks (MOFs) are highly promising porous materials in which metal ions or oxoclusters are interconnected by organic linkers. The large variety of organic linkers and metallic nodes enables the design and synthesis of many MOF structures with different intrinsic features, such as pore sizes, shapes, surface areas, and functions. ${ }^{1}$ The highly tailorable nature of MOFs shows great potential in a variety of applications such as gas storage and separation, ${ }^{2}$ chemical sensors,${ }^{3}$ (photo)catalysis, ${ }^{4,5}$ and others. ${ }^{6,7}$ In recent years, heterogeneous catalysts with biomimetic features, also called nanozymes, have been developed based on MOFs, nanoparticles, and nanowires. ${ }^{8}$ Biomimetic MOF-based catalysts can be obtained by either using MOF's intrinsic reactivity to catalyse the biological reactions through an active site on the metal cluster (e.g., a $\mathrm{Ce}^{\mathrm{III}} / \mathrm{Ce}^{\mathrm{IV}}$ redox active couple) or the organic linkers (e.g., (metallo)porphyrins) or by enzyme immobilisation into the MOF structure. ${ }^{9-11}$ In general, these approaches have resulted in MOF catalysts exhibiting oxidase, peroxidase, catalase or superoxide dismutase activities. ${ }^{8,10,12,13}$ Unlike widely explored redox activity, development of heterogeneous nanozymes with hydrolytic activity has received much less attention, and has been largely focused on the hydrolysis of phosphoester bonds in nerve agents. ${ }^{14,15}$

Despite its potential for a variety of applications in proteomics and biotechnology, ${ }^{16}$ the hydrolysis of peptide bonds by MOF-based nanozymes has been scarcely addressed. ${ }^{17-20}$ The area of proteomics largely relies on the hydrolysis of peptide bond by trypsin due to its high selectivity, high activity and broad availability. However, trypsin cleaves the C-terminal bond of the highly prevalent lysine and arginine residues, and typically results in many peptide fragments that are too short ( $\leq 6$ amino acids) to be unambiguously analysed by mass spectrometric techniques. ${ }^{21}$ This often results in an incomplete protein sequence coverage and subsequent loss of information. ${ }^{21,22}$ In this context, the presence of metal ions in the active sites of natural hydrolases sparked the development of synthetic metal complexes capable of peptide bond hydrolysis. ${ }^{23,24}$ Early studies developed mainly homogeneous complexes of metals like $\mathrm{Cu}^{\mathrm{II}},{ }^{25,26} \mathrm{Zr}^{\mathrm{IV}},{ }^{27} \mathrm{Pd}^{\mathrm{II}},{ }^{23,28}$ and $\mathrm{Pt}^{\mathrm{II} 23,28}$ as hydrolytic agents. Despite providing important insights into the 
catalytic mechanism of peptide bond hydrolysis, these complexes often suffered from restrictive reaction conditions and/or difficult separation from the produced peptides, which is an important step for smooth downstream analysis. Alternatively, heterogenous $\mathrm{Zr}_{6} \mathrm{O}_{8}$-based MOFs materials ( $\mathrm{Zr}$-MOFs) have recently emerged as promising proteolytic agents, given their nanozymatic hydrolytic activity and the ease of catalyst/protein digest separation. ${ }^{16}$ More specifically, the hydrolytic activity of Zr-based MOF-808, ${ }^{18}$ UiO- $66{ }^{19}$ and NU-1000, ${ }^{20}$ has been shown to greatly accelerate peptide bond cleavage in simple dipeptides and protein substrates at physiological $\mathrm{pH}$ conditions, in comparison to the uncatalyzed reaction. Furthermore, MOF structural features such as the connectivity of the metallic node, pore size and shape, and ligand decoration have been observed to directly impact the hydrolytic activity, strongly suggesting that tuning of MOF structure can boost MOF nanozymatic activity and selectivity toward peptide bonds. ${ }^{18-}$ 20

In contrast with the more developed $\mathrm{Zr}$-based artificial proteases, and in spite of the similar chemical properties of $\mathrm{Zr}^{\mathrm{IV}}$ and $\mathrm{Hf}^{\mathrm{IV}}$, the proteolytic potential of $\mathrm{Hf}^{\mathrm{IV}}$ complexes have been scarcely explored with only a few examples of soluble $\mathrm{Hf}(\mathrm{IV})$-substituted polyoxometalates (POMs) reported as a promising strategy for protein hydrolysis. ${ }^{29,30}$ Recently we demonstrated that an insoluble octadecanuclear Hf oxocluster, $\left[\mathrm{Hf}_{18} \mathrm{O}_{10}(\mathrm{OH})_{26}\left(\mathrm{SO}_{4}\right)_{13}\left(\mathrm{H}_{2} \mathrm{O}\right)_{33}\right]\left(\mathbf{H f}_{18}\right)$, cleaves myoglobin with a remarkable Asp-X/X-Asp siteselectivity. Mechanistic studies suggested the synergy between the Lewis and Bronsted acidity of the $\mathbf{H f}_{\mathbf{1 8}}$ cluster plays a key role in the hydrolysis selectivity. ${ }^{31}$ Interestingly, a similar contribution of the Bronsted acidity has been reported to boost the reactivity of a $\mathrm{Hf}_{6} \mathrm{O}_{8}$-based NU-1000 (Hf-NU-1000) in comparison to an analogous $\mathrm{Zr}-\mathrm{NU}-1000$ towards the chemical fixation of $\mathrm{CO}_{2}$ into five-membered cyclic carbonates. ${ }^{32}$ Intrigued by this unique dual Lewis/Bronsted acidity of $\mathrm{Hf}(\mathrm{IV})$ oxo-clusters which seem to directly impacts the reactivity, and the potential of Zr-MOF materials as heterogeneous proteolytic agents, in this work we set out to develop the proteolytic activity of the Hf-NU-1000 MOF given that $\mathrm{Hf}_{6} \mathrm{O}_{8}$-based MOFs (Hf-MOFs) have been much less explored in catalysis than their $\mathrm{Zr}$ counterparts. ${ }^{33-35}$ In contrast to the discrete nature of $\mathbf{H f}_{\mathbf{1 8}}$ cluster we studied previously, the $\mathrm{Hf}$ oxo-clusters $\left[\mathrm{Hf}_{6}\left(\mu_{3}-\mathrm{O}\right)_{4}\left(\mu_{3}-\right.\right.$ $\left.\mathrm{OH})_{4}\left(\mathrm{H}_{2} \mathrm{O}\right)_{4}(\mathrm{OH})_{4}\right]^{8+}$ which constitute Hf-NU-1000 MOF are connected by organic ligands resulting in the extended porous material. Thus, in addition to further developing Hf-based nanozymes as site-selective proteolytic agents, this study provides comparative insights into the nanozymatic activities of discrete and MOF-embedded Hf oxo-clusters. Moreover, given our previous study with $\mathrm{Zr}-\mathrm{NU}-1000 \mathrm{MOF},{ }^{20}$ it also probes the potential of a Hf-MOF to provide a better hydrolytic profile than its $\mathrm{Zr}$ analogue. 


\section{Results and discussion}

To explore the viability of Hf oxo-clusters when embedded in a porous material as artificial proteolytic agents, we have selected the NU-1000 topology as a representative MOF structure given its high similarity to the Zr-NU-1000 MOF studied previously (Figure 1). ${ }^{20}$ The Hf-NU-1000 consists of hexameric Hf oxoclusters $\left[\mathrm{Hf}_{6}\left(\mu_{3}-\mathrm{O}\right)_{4}\left(\mu_{3}-\mathrm{OH}\right)_{4}\left(\mathrm{H}_{2} \mathrm{O}\right)_{4}(\mathrm{OH})_{4}\right]^{8+}$ that are 8 -connected by large 1,3,6,8-( $p$-benzoate)pyrene (TBAPy ${ }^{4-}$ ) linkers, which results in a csq network with hexagonal mesopores (31 $\left.\mathrm{A}\right)$ and triangular $12 \AA$ micropores. ${ }^{32,36}$ The synthesis of Hf-NU-1000 was performed as previously reported, ${ }^{32}$ and the structure of the resulting MOF was confirmed by powder X-ray diffraction (PXRD) (Figure S6) and scanning electron microscopy (SEM) (Figure 1B-C). Interestingly, SEM indicated that the particles were twice as large (around $10 \mu \mathrm{m}$ ) in comparison to original report. ${ }^{32}$
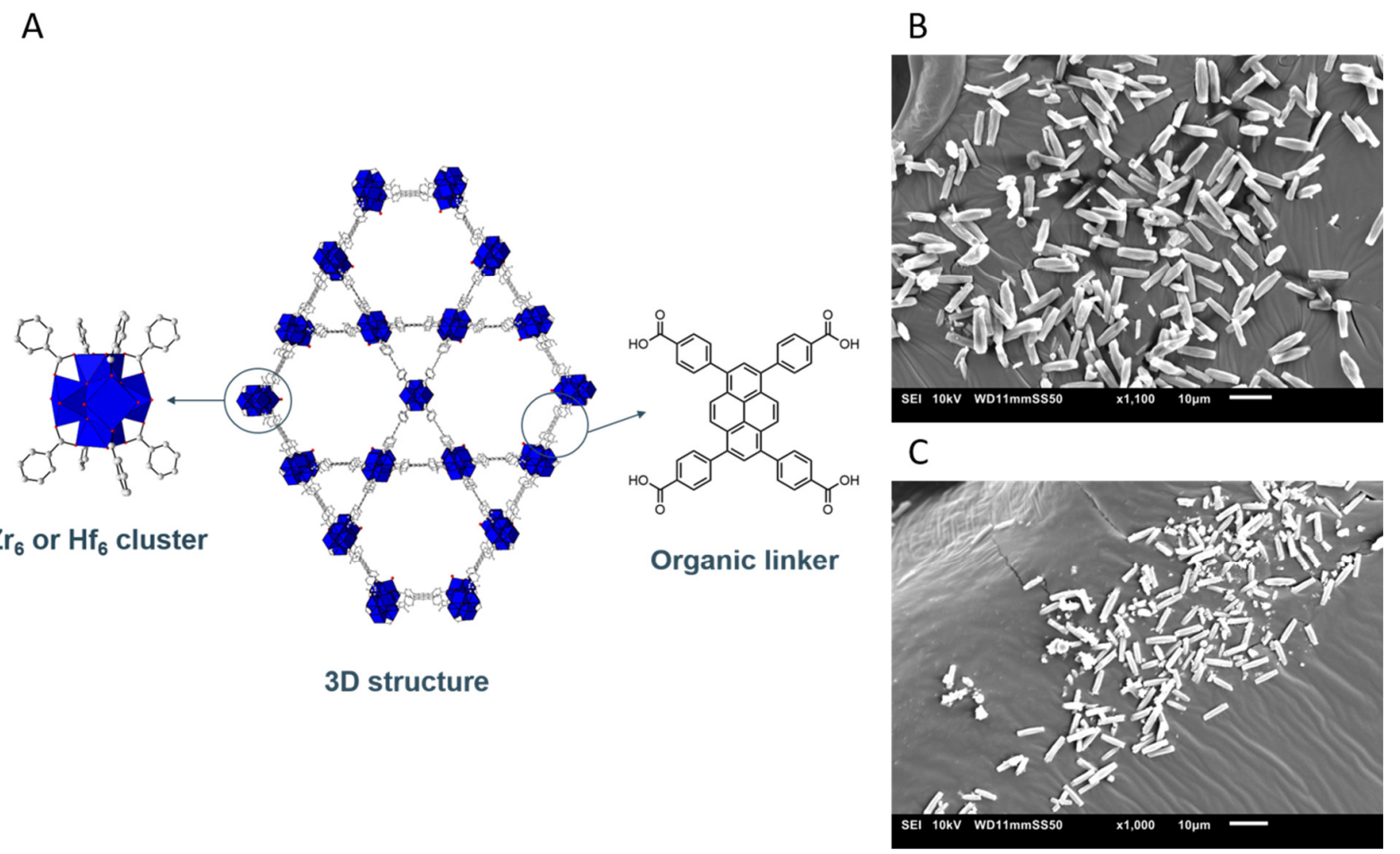

Figure 1. A) Representation of the Hf/Zr-NU-1000 structure highlighting the corresponding cluster node and linker units. $\mathrm{Zr}_{6} / \mathrm{Hf}_{6}$ cluster $=\left[\mathrm{Zr}_{6} / \mathrm{Hf}_{6}\left(\mu_{3}-\mathrm{O}\right)_{4}\left(\mu_{3}-\mathrm{OH}\right)_{4}\left(\mathrm{H}_{2} \mathrm{O}\right)_{4}(\mathrm{OH})_{4}\right]^{8+}$. B) SEM image of Hf-NU1000 as synthesized (after activation). C) SEM image of Hf-NU-1000 after 24h incubation with GG at $60{ }^{\circ} \mathrm{C}$. 


\section{Hydrolytic activity of Hf-NU-1000 toward peptide bond hydrolysis}

Following our previous work, ${ }^{18-20}$ the hydrolytic activity of Hf-NU-1000 was initially screened by using glycylglycine (GG) as model substrate. The hydrolytic cleavage of GG was conveniently monitored by ${ }^{1} \mathrm{H}$ NMR spectroscopy, which indicated formation of 2 equivalents of glycine, and the cyclization of GG into cyclic glycylglycine (cGG), which is a side-product formed through an intramolecular condensation of the carboxylate- and amino-terminal groups of GG (Figure S1). Upon incubation of equimolar amounts of GG and Hf-NU-1000 at pD 7.4 and $60{ }^{\circ} \mathrm{C}$, the decrease of GG concentration was followed over time, plotted as a function of time (Figure 2a) and fitted to a first order exponential decay (Figure 2b). The fitted data resulted in a rate constant of $\mathrm{k}_{\mathrm{obs}}=8.33 \times 10^{-7} \mathrm{~s}^{-1}$ at $60^{\circ} \mathrm{C}$ and $\mathrm{pD} 7.4$, which corresponds to a half-life $\left(\mathrm{t}_{1 / 2}\right)$ of 231 hours representing a significant acceleration compared to the uncatalyzed reaction $\left(\mathrm{t}_{1 / 2}=6\right.$ years at $60{ }^{\circ} \mathrm{C}$ and $\left.\mathrm{pH} 7\right) .{ }^{37}$ In addition, catalytic turnover was observed when incubating a tenfold excess of GG $(20 \mu \mathrm{mol})$ with Hf-NU-1000 $(2 \mu \mathrm{mol})$. An almost complete hydrolysis of the peptide bond was obtained after $505 \mathrm{~h}$ with a rate constant of $8.94 \times 10^{-7} \mathrm{~s}^{-1}\left(\mathrm{t}_{1 / 2}=215 \mathrm{~h}\right)$ indicating that Hf-NU1000 acts as a catalyst for the peptide bond hydrolysis (TON = 9) (Figure S2). Notably, this reactivity is already strikingly different from the one previously observed with the $\mathbf{H f}_{\mathbf{1 8}}$ cluster, ${ }^{31}$ since $\mathbf{H} \mathbf{f}_{\mathbf{1 8}}$ strongly adsorbed to peptides and proteins, hindering the evaluation of dipeptide hydrolysis. These results indicate that when the Hf oxo-cluster is embedded in a framework such adsorption is greatly decreased, enabling the reactivity to be observed in rather smooth conditions. 
A

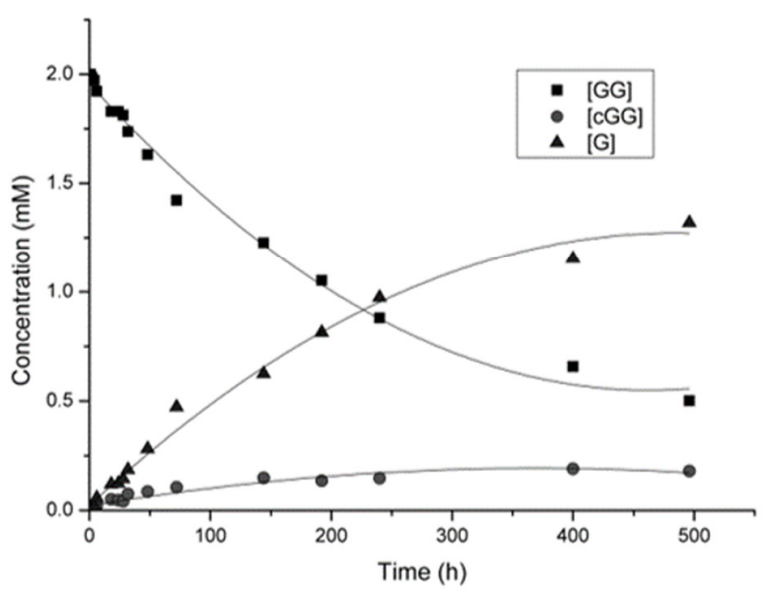

B

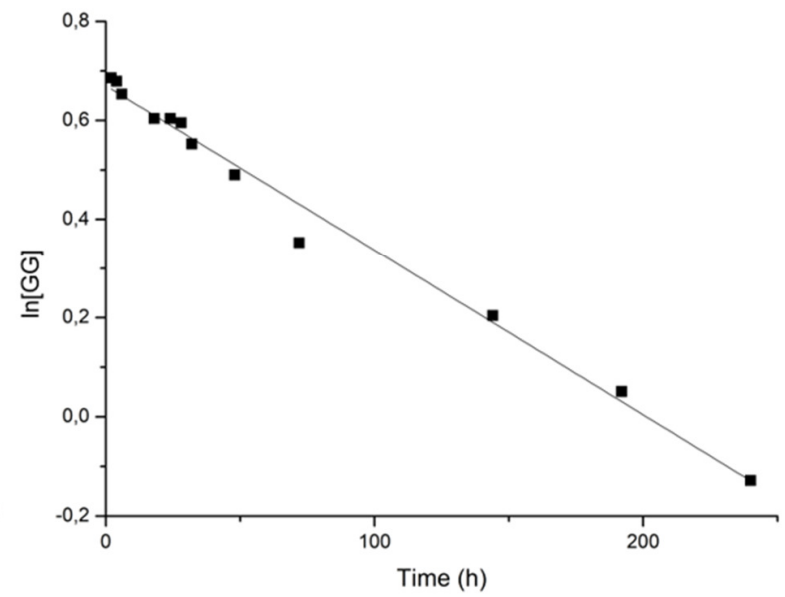

Figure 2. A) Concentration profile of glycylglycine (GG), cyclic glycylglycine (cGG) and glycine (G) over time in presence of $2 \mu \mathrm{mol} \mathrm{Hf-NU1000} \mathrm{at} 60{ }^{\circ} \mathrm{C}$ and pD 7.4. B) First order decay fit of $\ln [\mathrm{GG}]$ as a function of time for $2 \mathrm{mM} \mathrm{GG}$ in presence of $2 \mu \mathrm{mol}$ Hf-NU-1000 at $60{ }^{\circ} \mathrm{C}$ and $\mathrm{pD}$ 7.4. $\left(\mathrm{R}^{2}=0.98788\right)$.

Giving the relevance of Bronsted acidity attributed for the reactivity of Hf-based materials in previous reports, ${ }^{31,32}$ the effect of $\mathrm{pH}$ in the hydrolytic activity of Hf-NU-1000 was further investigated. Using identical temperature and substrate concentrations to the initial screening conditions, we followed the GG hydrolysis in solutions with pD values ranging from 5.4 to 9.4. Similar to Zr-NU-1000, the highest reaction rates were observed in the pD range between 7.5 and 8.5 (Figure S3). Notably, in this range the carboxylate group of GG is deprotonated, allowing for an efficient substrate-metal node interaction and a swift deprotonation of the entering water molecule. Such mechanism has been previously theoretically predicted for an analogous Zr-MOF, ${ }^{38}$ and could explain the higher activity in mildly alkaline solutions. The observed lower adsorptions of GG at lower $\mathrm{pH}$ values indicates that the substrate-material interaction is less efficient, which is consistent with the lower rates observed in mildly acidic solutions (Figure S3). Most interestingly, the higher activity of Hf-NU-1000 at neutral and mildly alkaline pD values is the

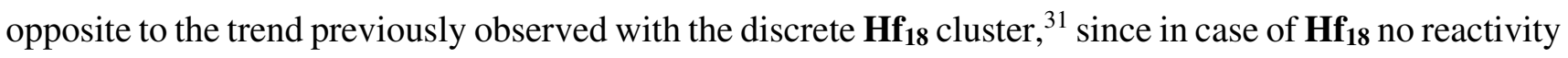
was observed when the reaction was carried out at $\mathrm{pD}$ 7.4. This suggests that Hf oxo-cluster reactivity toward peptide bond is rather distinct when they represent discrete entities in the reaction or when they are embedded into an extended porous material. Presumably, in the case of Hf-NU-1000 a Lewis acid catalytic mechanism is most likely the major one since the rates are higher in slightly alkaline solutions. This hypothesis is fully in line with our recent mechanistic studies. ${ }^{38}$ 
The temperature also exerted a strong effect on the observed reaction rate, and calculation of activation parameters evidenced a large entropic contribution to the energy barrier, as observed before for the NU1000 topology in these reactions. ${ }^{20}$ The activity of Hf-NU-1000 was strongly affected by an increase in the reaction temperature with nearly 20 -fold reduction to the half-life from 990 hours at $40^{\circ} \mathrm{C}$ to 51 hours at $80{ }^{\circ} \mathrm{C}$. Furthermore, by fitting the reaction rates to the Arrhenius equation, the apparent activation energy $\left(E_{\text {act }}\right.$ ) of $62 \mathrm{~kJ} \mathrm{~mol}^{-1}$ could be obtained for Hf-NU-1000 (Figure S4-Figure S5). Additionally, the enthalpy $\left(\Delta H^{\ddagger}=60 \mathrm{~kJ} \mathrm{~mol}^{-1}\right)$ and entropy of activation $\left(\Delta S^{\ddagger}=-183 \mathrm{~J} \mathrm{~mol}^{-1} \mathrm{~K}^{-1}\right)$ of the reaction of mediated by Hf-NU-1000 were calculated by linear fitting of $\ln (\mathrm{k} / \mathrm{T})$ as a function of $1 / \mathrm{T}$ (Figure S6). Based on the enthalpy and entropy, the Gibbs energy of activation at $37{ }^{\circ} \mathrm{C}$ was determined to be $117 \mathrm{~kJ} \mathrm{~mol}^{-1}$.

\section{Hydrolytic activity of Hf-NU-1000 toward Gly-X substrates}

In addition to GG, a series of other Gly-X dipeptides (X = Ser, Asp, Leu, Tyr, Ile, Phe, Ala, Asn, Lys and His) was incubated with Hf-NU-1000 to investigate the potential influence of side chain functional groups and steric hindrance on the reactivity (Figure 3). As expected, we observed complete adsorption of GlyTyr and Gly-Phe peptides, most likely due to the strong potential for $\pi-\pi$ stacking of the phenyl-based Tyr and Phe side chains with the aromatic MOF linker. Interestingly, two dipeptides, Gly-Ser and GlyHis, showed higher conversion rates than GG under similar conditions, suggesting that side chains containing groups that are able to enhance the interaction with the $\mathrm{Hf}_{6}$ cluster may facilitate the overall reaction. Alternatively, the superior rates observed for Gly-Ser can also be attributed to a N,O-acyl rearrangement prior to hydrolysis. ${ }^{39,40}$ A similar mechanism might explain the more efficient hydrolysis of Gly-Asp in comparison to Gly-Asn. ${ }^{31}$ The absence of hydrolysis observed for Gly-Asn and Gly-Lys evidences that not all groups that can coordinate to the cluster lead to productive intermediates, and may also contribute to slow the overall reaction. In addition, the $\mathrm{NH}_{2}$ group in the side chain of Gly-Lys is positively charged at $\mathrm{pH} 7.4$, which might have inhibited the substrate-MOF interaction. Interestingly, the negative charge of Gly-Asp side chain did not hamper the reactivity, indicating that charge can play a role in the hydrolysis. Previous hydrolysis of Gly-Asn and Gly-Lys by MOF- $808^{18}$ and UiO-66 ${ }^{19}$ MOFs suggests MOF structure plays a key role in the peptidase activity, and that selectivity can be achieved by tuning structural feature such as metal composition and/or topology of MOF materials. Finally, the similar conversion observed for Gly-Gly, Gly-Ala, Gly-Asp and Gly-Ile despite their distinct sizes suggests the reaction is much less sensitive to the steric hindrance of the $\mathrm{X}$ side chain than observed previously with Zr-based MOF-808 and UiO-66 MOFs. ${ }^{18,19}$ Nevertheless, no hydrolysis was observed for Gly-Leu. The absence of hydrolysis for Gly-Leu while Gly-Ile reacted as other substrates is not fully understood yet. 
However, it further showcases a high level of selectivity able to differentiate very similar groups might arise from fine tuning the structure of MOF catalysts.

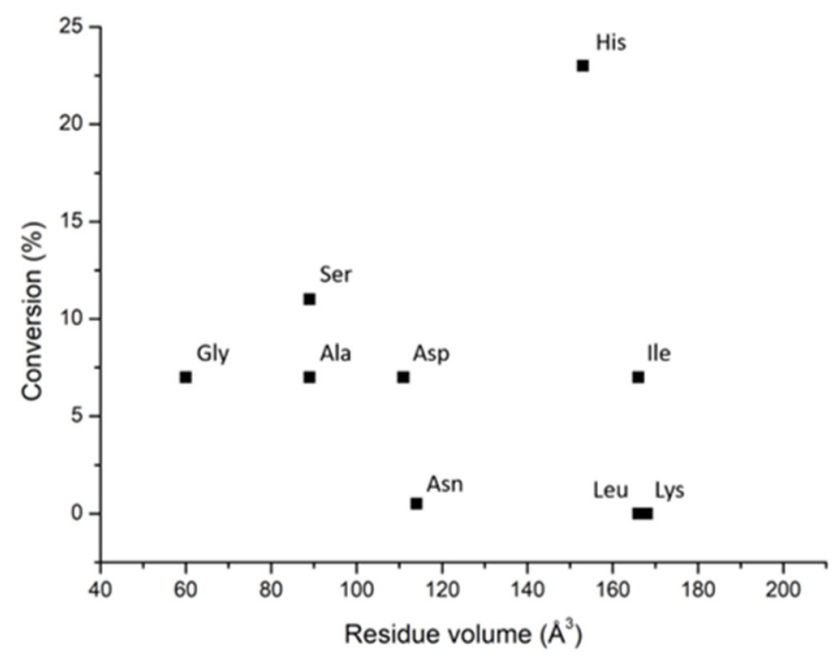

Figure 3. Conversion of Gly-X dipeptides by Hf-NU-1000 at $60{ }^{\circ} \mathrm{C}$ at $\mathrm{pH} 7.4$ after $24 \mathrm{~h}$, amino acid residue volumes based on reported values. ${ }^{41}$

\section{Hydrolytic activity of Hf-NU-1000 toward protein hydrolysis}

The hydrolytic potential of Hf-NU-1000 towards a larger substrate, hen egg white lysozyme (HEWL), was also evaluated. To this end, HEWL was incubated with Hf-NU-1000 at $60{ }^{\circ} \mathrm{C}$ and pD 7.0, mimicking the reaction with Zr-NU-1000 previously reported. ${ }^{20}$ Sodium dodecyl sulphate polyacrylamide gel electrophoresis (SDS-PAGE) analysis of the reaction at 5, 24 and $72 \mathrm{~h}$ intervals clearly evidenced that only 3 protein fragments were formed with an estimated molecular weight of 12.3, 10.1 and 8.4 kDa, which are not present in the control experiment (lanes 1-3 vs. 8 in Figure 4). A comparison of Zr- and HfNU-1000 MOF proteolytic activity through analysis of protein digests in the same SDS-PAGE electropherogram, which allows a side-by-side comparison with lower experimental variance, confirmed the cleavage pattern for Hf- and Zr-NU-1000 is identical (lanes 1-3 vs. 4-5, respectively, in Figure 4). The estimated molecular weight of the fragments observed for Hf-NU-1000, 12.4, 10.1 and $8.2 \mathrm{kDa}$, and for $\mathrm{Zr}-\mathrm{NU}-1000,12.2,10.0$, and $8.3 \mathrm{kDa}$, are identical within the error. Together, this data suggests that Hf-NU-1000 might also be cleaving HEWL at Asp-X/X-Asp peptide bonds, as deduced previously for the Zr-NU-1000. ${ }^{20}$ Interestingly, $\mathbf{H f}_{18}$ cluster has cleaved myoglobin selectively at Asp-X/X-Asp sites, suggesting that Hf oxo-clusters exhibit a similar selectivity regardless if used as discrete units or embedded in extended porous materials. 


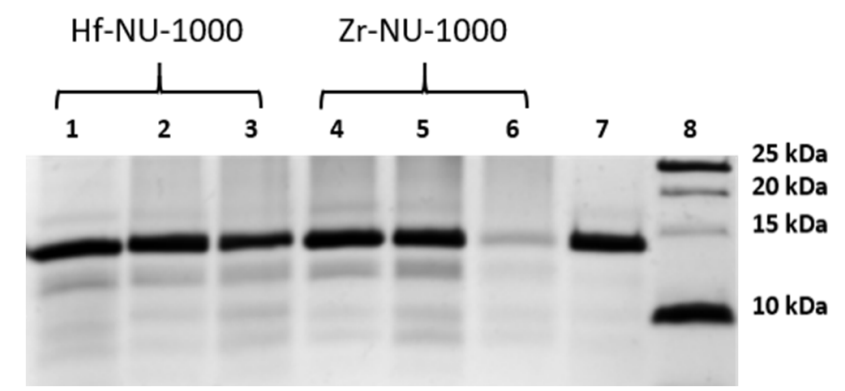

Figure 4. Silver-stained SDS-PAGE analysis of HEWL in presence of Hf-NU-1000 (lanes 1-3) and ZrNU-1000 (lanes 4-6). Lane 1, 2 and 3 show HEWL in presence of Hf-NU-1000 with, respectively, 5 hours, 24 hours and 72 hours of incubation time. Lane 4, 5 and 6 show HEWL in presence of Zr-NU-1000 with, respectively, 5 hours, 24 hours and 72 hours of incubation time. Lane 7 is HEWL as a control sample after 72 hours of incubation in absence of MOF. Lane 8 is the protein ladder.

\section{Stability of Hf-NU-1000 during hydrolysis reaction}

SEM analysis after 24 hours of reaction with $\mathrm{GG}$ at $60^{\circ} \mathrm{C}$ showed that the original particles retained their size (Figure 1), indicating that Hf-NU-1000 is stable under reaction conditions. Further, Hf-NU-1000 samples incubated with GG in solutions with $\mathrm{pH}$ values ranging from 3.0 to 9.0 were analysed by PXRD (Figure S7). After 24 hours, the overall structure stability of Hf-NU-1000 was retained at all pH values. Finally, TGA analysis confirmed that Hf-NU-1000 conserves its overall temperature stability after reactions with GG and HEWL (Figure S8).

The hydrolysis of peptide bonds by Hf-NU-1000 is heterogeneous in nature. After incubation of GG with the Hf-NU-1000 for 2 days at $60^{\circ} \mathrm{C}$, the MOF was removed by filtration and the supernatant was kept at $60{ }^{\circ} \mathrm{C}$ for 14 days. After removal of the MOF, the GG hydrolysis did not proceed further, suggesting that the hydrolysis required the presence of the solid MOF material and is not due to leached $\mathrm{Hf}^{4+}$ ions. Furthermore, ICP-OES experiments showed that very low levels of $\mathrm{Hf}^{4+}$ ions $(0.80( \pm 0.003) \mathrm{ppm}$, corresponding to $0.04( \pm 0.003) \%$ of initial Hf-NU-1000) could be detected after 24 hours of incubation at $60{ }^{\circ} \mathrm{C}$.

The structural stability under reaction conditions, and the heterogeneous nature of the reaction prompted us to probe the recyclability of the material. The recyclability of Hf-NU-1000 was evaluated after separating Hf-NU-1000 and GG by centrifugation after 24 hours of incubation at pD 7.4 and $60{ }^{\circ} \mathrm{C}$. The MOF was then washed overnight with $\mathrm{D}_{2} \mathrm{O}$ to ensure removal of residual substrate. After drying the MOF 
under air, a new portion of GG substrate was incubated with the recovered material under the same conditions. The hydrolysis rates of GG to G and PXRD both showed stable measurements over the 5 of such reaction cycles (Figure 5A-B), thereby showcasing excellent recyclability of Hf-NU-1000 .

A
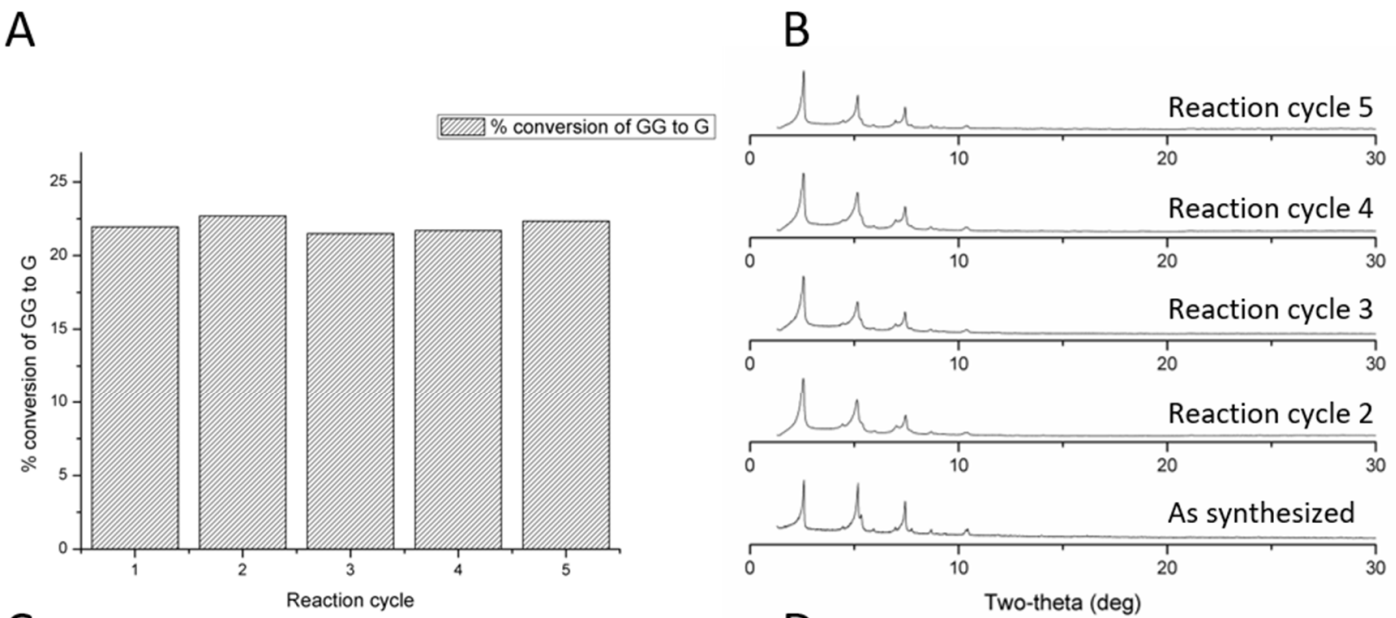

C

$\mathrm{D}$
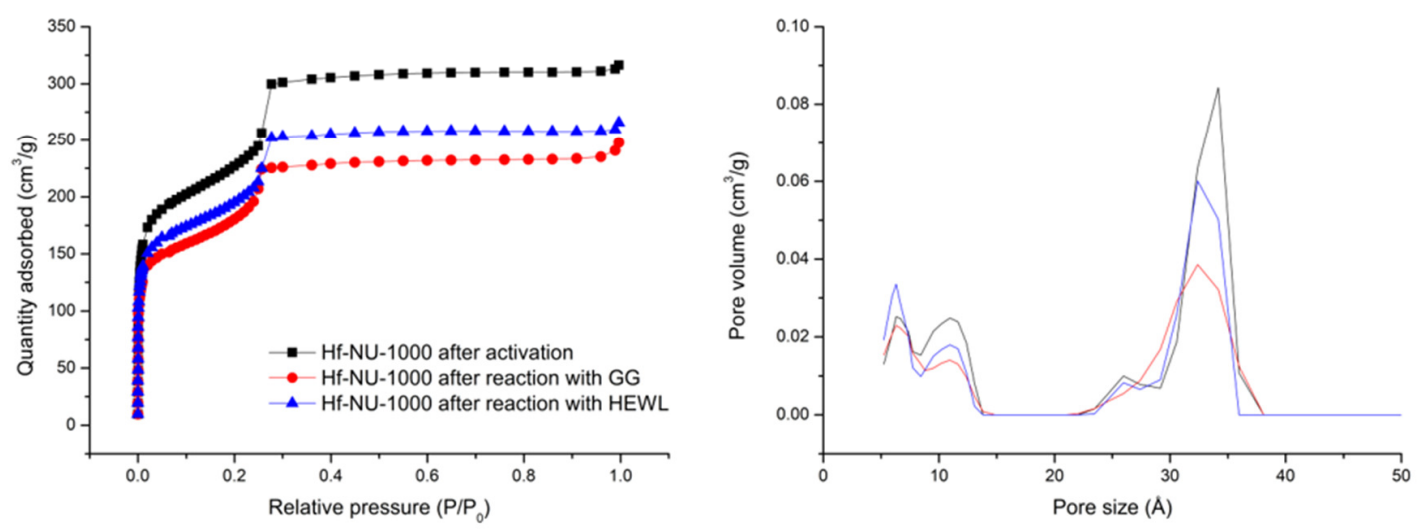

Figure 5. A) Conversion of $2 \mathrm{mM} \mathrm{GG}$ to $\mathrm{G}$ over 5 reaction cycles in presence of $2 \mu \mathrm{mol} \mathrm{Hf}-\mathrm{NU}-1000$ at $60{ }^{\circ} \mathrm{C}$ and pD 7.4. B) PXRD patterns of $2 \mu \mathrm{mol}$ Hf-NU-1000 after each hydrolysis reaction cycle, and solvent exchange with $\mathrm{D}_{2} \mathrm{O}$ overnight. C) $\mathrm{N}_{2}$ isotherm of Hf-NU-1000 as synthesized (black), after reaction with $2 \mathrm{mM} \mathrm{GG}\left(60{ }^{\circ} \mathrm{C}, \mathrm{pH} 7.0,24 \mathrm{~h}\right)$ (red), and after reaction with $0.02 \mathrm{mM}$ HEWL $\left(60{ }^{\circ} \mathrm{C}, \mathrm{pH}\right.$ 7.0, 24 h) (blue). D): Pore size distribution of Hf-NU-1000 as synthesized (black), after reaction with 2 $\mathrm{mM} \mathrm{GG}\left(60^{\circ} \mathrm{C}, \mathrm{pH} 7.0,24 \mathrm{~h}\right)$ (red), and after reaction with $0.02 \mathrm{mM}$ HEWL $\left(60^{\circ} \mathrm{C}, \mathrm{pH} 7.0,24 \mathrm{~h}\right)($ blue $)$.

Finally, considering the more suitable adsorption profile of Hf-NU-1000 in comparison to the $\mathbf{H} \mathbf{f}_{\mathbf{1 8}}$, we further investigated the adsorption of substrates to the Hf-NU-1000 material. The adsorption of increasing concentrations of GG solutions by Hf-NU-1000 was followed at different $\mathrm{pH}$ values $(3.4,5.4$ or 7.4) by 
incubations for 6 hours to ensure equilibrium was reached. Additionally, to avoid hydrolysis during the adsorption assay, incubation was carried out at room temperature. The amount of adsorption was followed by ${ }^{1} \mathrm{H}$ NMR spectroscopy and the data depicted in Figure 6. Interestingly, the higher reaction rates at $\mathrm{pH}$ values around 7 coincided with higher adsorption rates at this $\mathrm{pH}$. These NMR results are also consistent with $\mathrm{N}_{2}$ physisorption (Figure 5C) studies, which revealed a $\sim 20 \%$ reduction in BET surface area before $\left(837 \mathrm{~m}^{2} \mathrm{~g}^{-1}\right)$ and after (GG: $658 \mathrm{~m}^{2} \mathrm{~g}^{-1}$ ) the GG hydrolysis reactions. For the hydrolysis of HEWL, the reduction in BET was slightly lower $\left(\sim 16 \%, 721 \mathrm{~m}^{2} \mathrm{~g}^{-1}\right)$, presumably because of the large size of this protein. Interestingly, the pore size distribution did not change upon adsorption of the substrates, suggesting that the impact on the overall structure of Hf-NU-1000 was minimal (Figure 5D), consistent with the stability and recyclability results mentioned above.

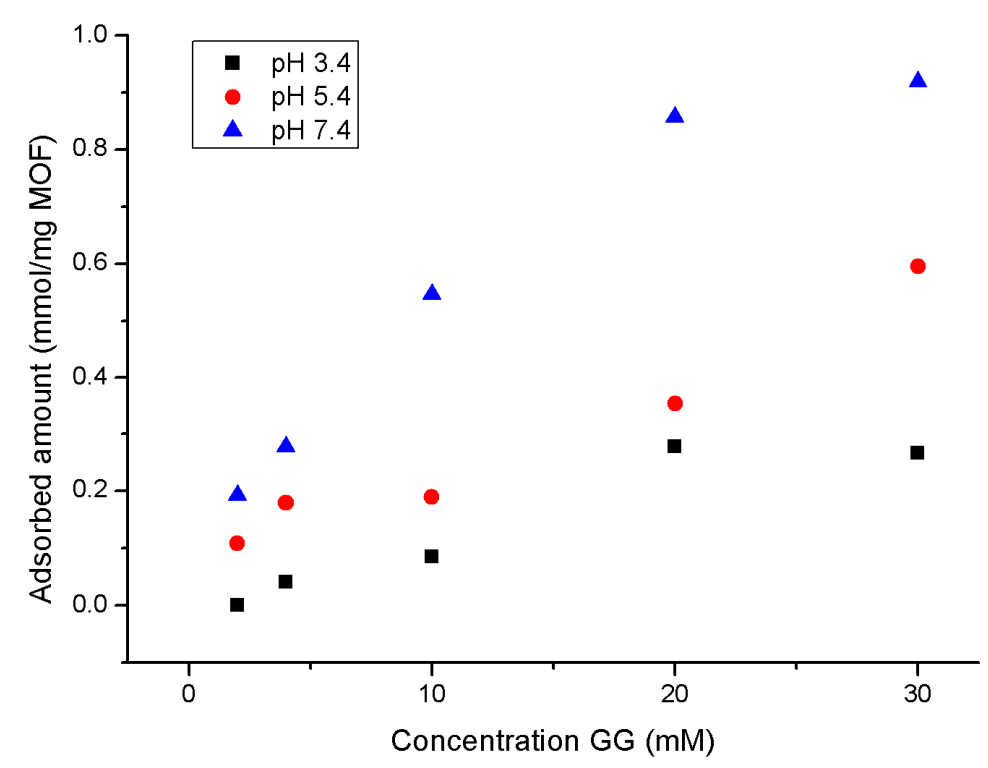

Figure 6. Adsorption of GG by Hf-NU-1000 as a function of concentration at pH 3.4, 5.4 and 7.4 at room temperature for 6 hours.

\section{Comparative analysis of Hf-NU-1000 catalytic activity}

In comparison with our previous report on the proteolytic potential of $\left[\mathrm{Hf}_{18} \mathrm{O}_{10}(\mathrm{OH})_{26}\left(\mathrm{SO}_{4}\right)_{13}\left(\mathrm{H}_{2} \mathrm{O}\right)_{33}\right]$ (Hf $\mathbf{H}_{18}$ ), a discrete and heterogeneous $\mathrm{Hf}$ oxo-cluster, our results strongly suggests that incorporation of $\mathrm{Hf}$ oxo-clusters in porous frameworks greatly enhance their hydrolytic profile, enabling the hydrolysis of several dipeptides and a protein under mild conditions. The adsorption studies performed in this work indicate that having the Hf oxo-cluster catalytic units embedded in a network decreases the overall 
adsorption of substrates which was observed with Hf 18 cluster. Comparatively, Hf-NU-1000 also performed better in terms of adsorption when compared to Zr-NU-1000, for which greater proportional reduction of surface was observed after the reaction. Additionally, unlike the $\mathbf{H f}_{\mathbf{1 8}}$ cluster that required special elution protocols to analyse the protein digest by SDS-PAGE, the Hf-NU-1000/protein mixture could be directly analysed through a standard procedure. More interestingly, the pore size distribution remained unchanged after the reaction for Hf-NU-1000, while a $\sim 15 \%$ pore size reduction was observed for Zr-NU-1000 after hydrolysis of HEWL. This suggests the effect of protein adsorption on the overall MOF structure was significantly lower for Hf-NU-1000.

Besides adsorption, Hf-NU-1000 reactivity profile also presented important differences in comparison with previous reports. As Zr- and Hf-NU-1000 MOFs are isostructural, a straightforward comparison of both MOFs can be made. The difference in hydrolysis rate between Hf-NU-1000 and Zr-NU-1000 ${ }^{20}$ is depicted in Figure 7. Even though Hf-NU-1000 provides a significant acceleration of the hydrolysis of GG over the non-catalysed reaction, the reaction rates for Hf-NU-1000 are slightly but consistently lower than the rates observed for Zr-NU-1000, taking into account similar standard deviations in the Zr-NU1000 reaction rates. Previously, a small but potentially important difference in the $\mathrm{pK}_{\mathrm{a}}$ values of the $\mathrm{Zr}^{\mathrm{IV}}$ $\left(\mathrm{pK}_{\mathrm{a}}=4.59\right)$ and $\mathrm{Hf}^{\mathrm{IV}}$ MOFs $\left(\mathrm{pK}_{\mathrm{a}}=4.44\right)$ was reported. ${ }^{32}$ However, given that the rate of hydrolysis for both materials peak in the $\mathrm{pH}$ range of $7-8$, such small difference in intrinsic Bronsted acidity likely plays a minor, if any, role in this reactivity difference. On the other hand, the energy barrier for the Hfand Zr-NU-1000 hydrolysis reactions derive from slightly different enthalpy and entropy contributions in each case (Hf-NU-1000: $\Delta H^{\ddagger}=60 \mathrm{~kJ} \mathrm{~mol}^{-1}$ and $\Delta S^{\ddagger}=-183 \mathrm{~J} \mathrm{~mol}^{-1} \mathrm{~K}^{-1} ; \mathrm{Zr}-\mathrm{NU}-1000: \Delta H^{\ddagger}=49 \mathrm{~kJ} \mathrm{~mol}^{-1}$ and $-221 \mathrm{~J} \mathrm{~mol}^{-1} \mathrm{~K}^{-1}$ ). Arguably, the higher enthalpy of activation for Hf-NU-1000 entails from the stronger $\mathrm{Hf}-\mathrm{O}$ bond in comparison to $\mathrm{Zr}-\mathrm{O}$ one, ${ }^{32}$ which could slow down ligand exchange steps and result in slower rates. However, both Hf- and Zr-NU-1000 reactions have the same $\Delta G^{\ddagger}(117 \mathrm{vs} 118 \mathrm{~kJ}$ $\mathrm{mol}^{-1}$ at $37^{\circ} \mathrm{C}$, respectively), suggesting that the observed reactivity difference stems from structural features rather than differences in the intrinsic reactivity between these elements. Consistent with this hypothesis, the BET surface area for Hf-NU-1000 $\left(837 \mathrm{~m}^{2} \mathrm{~g}^{-1}\right)$ is significantly lower than Zr-NU-1000 $\left(2192 \mathrm{~m}^{2} \mathrm{~g}^{-1}\right)$, even though the pore structure is similar between two materials. 


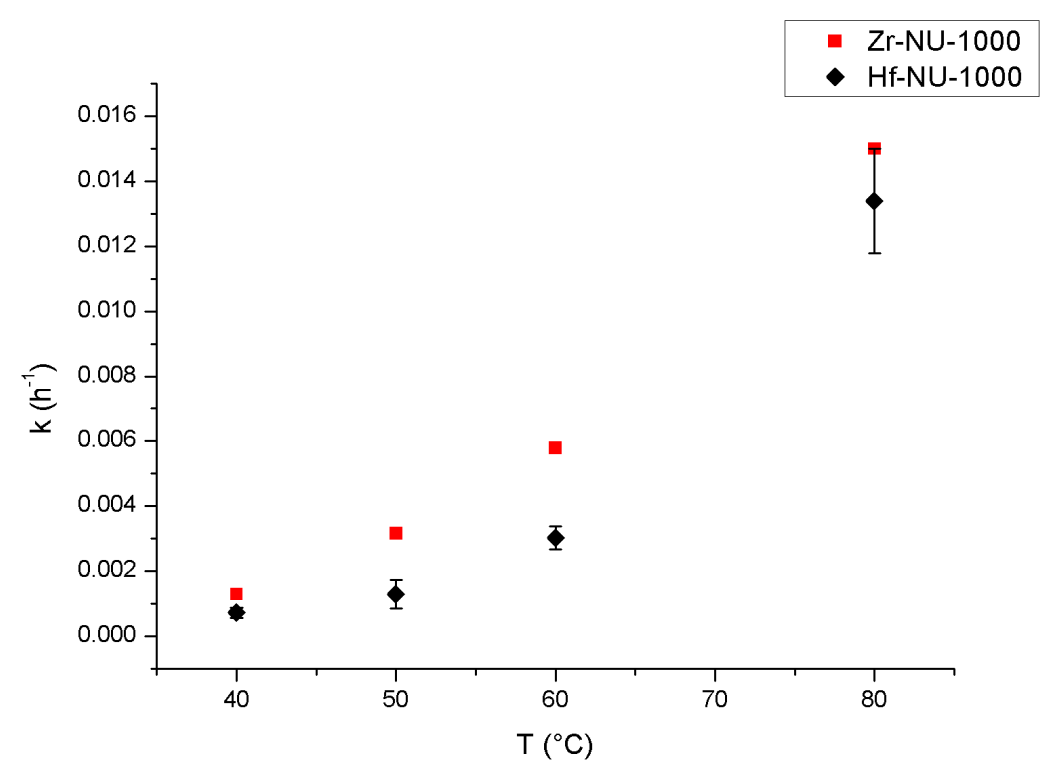

Figure 7. Graphical comparison of observed GG hydrolysis rates by Zr- and Hf-NU-1000 at different temperatures. Standard deviation of reaction rates of Hf-NU-1000 are based on 3 reaction replicates.

Despite the difference in reaction rates, both Hf-NU-1000 and Zr-NU-1000 present the same reactivity pattern towards HEWL protein, and both suggest a highly selective hydrolytic cleavage takes place. This level of selectivity resembles the one observed with $\mathbf{H f}_{\mathbf{1 8}}$ cluster which strongly suggests the main features of Hf oxo-clusters reactivity toward proteins are preserved when they are incorporated to a porous network like in Hf-NU-1000 MOF.

\section{Conclusion}

In summary, Hf-NU-1000 MOF smoothly hydrolyses peptide bonds in both dipeptides and proteins substrates under mild conditions. Using glycylglycine (GG) as a model substrate, catalytic turnover and an enhancement of four orders of magnitude compared to uncatalyzed reaction were observed. In addition, hydrolysis of other dipeptides revealed a lower influence of substrate size than the one observed for UiO66 and MOF-808 Zr-MOFs previously reported. In addition, Lewis basic group may accelerate or slow reactions in comparison to GG, presumably by additional coordination of side chain functional groups to the metallic node. These findings underline the possibility of developing a high level of peptide bond selectivity through additional design of MOF structure. More importantly, Hf-NU-1000 selectively hydrolyses HEWL protein, affording a cleavage pattern similar to the one observed previously with the 
isostructural Zr-NU-1000 MOF. These findings also show the immobilization of Hf oxo-clusters in a MOF structure imparts key differences in the reactivity observed with discrete $\mathbf{H f}_{\mathbf{1 8}}$ cluster studied previously while preserving its keen hydrolytic ability. For example, Hf oxo-clusters embedded in the NU-1000 structure exhibit higher reactivity at neutral $\mathrm{pH}$ and much weaker substrate adsorption, while $\mathbf{H f}_{\mathbf{1 8}}$ performs better in acidic conditions and remains strongly attached to products after the reaction. Consequently, embedding Hf oxo-clusters in the NU-1000 structure enabled catalytic turnover, hydrolysis of small dipeptide structures, and catalyst recycling to be observed. Moreover, weaker adsorption of HEWL protein streamlined analysis of protein digest by SDS-PAGE. In a broader context, these results enhance the prospect of Hf-based materials to be developed as robust hydrolytic nanozymes for selective protein cleavage that can be applied in proteomic and related areas.

\section{Author Information}

Corresponding Author*E-mail: tatjana.vogt@kuleuven.be

ORCID

Francisco de Azambuja: orcid.org/0000-0002-5537-5411

Tatjana N. Parac-Vogt: orcid.org/0000-0002-6188-3957

\section{Acknowledgements}

We thank KU Leuven (C14/19/076) and Research Foundation Flanders (FWO) for financial support (G0D3219N). A.L. (48730/1S10318N), C.S (68090/11C9320N), and F.d.A. (195931/1281921N) thank the FWO for fellowships. Authors thank Dr. S. Smolders for helping with TGA and $\mathrm{N}_{2}$ physisorption analysis.

\section{References}

1 H. Furukawa, K. E. Cordova, M. O’Keeffe and O. M. Yaghi, Science, 2013, 341, 1230444.

2 H. Li, K. Wang, Y. Sun, C. T. Lollar, J. Li and H. C. Zhou, Mater. Today, 2018, 21, 108-121.

3 L. E. Kreno, K. Leong, O. K. Farha, M. Allendorf, D. Van Richard P. and J. T. Hupp, Chem. Rev., 2012, 112, 1105-1125. 

$1450-1459$.

A. Dhakshinamoorthy, Z. Li and H. Garcia, Chem. Soc. Rev., 2018, 47, 8134-8172.

J. Liu, L. Chen, H. Cui, J. Zhang, L. Zhang and C. Y. Su, Chem. Soc. Rev., 2014, 43, 6011-6061.

A. Kirchon, L. Feng, H. F. Drake, E. A. Joseph and H. C. Zhou, Chem. Soc. Rev., 2018, 47, 8611-8638.

Y. Huang, J. Ren and X. Qu, Chem. Rev., 2019, 119, 4357-4412.

9

E. Gkaniatsou, C. Sicard, R. Ricoux, J. P. Mahy, N. Steunou and C. Serre, Mater. Horizons, 2017, 4, 55-63.

10

J. S. Qin, S. Yuan, C. Lollar, J. Pang, A. Alsalme and H. C. Zhou, Chem. Commun., 2018, 54, 4231-4249.

11 J. R. Bour, A. M. Wright, X. He and M. Dincă, Chem. Sci., 2020, 11, 1728-1737.

12 Y. Chen and S. Ma, Dalton Trans., 2016, 45, 9744-9753.

13 H. N. Abdelhamid, G. A. E. Mahmoud and W. Sharmoukh, J. Mater. Chem. B, 2020, 8, 7548-7556.

14 K. O. Kirlikovali, Z. Chen, T. Islamoglu, J. T. Hupp and O. K. Farha, ACS Appl. Mater. Interfaces, 2020, 12, 14702-14720.

A. Zammataro, R. Santonocito, A. Pappalardo and G. T. Sfrazzetto, Catalysts, 2020, 10, 1-18.

16

H. Zhong, Y. Li, Y. Huang and R. Zhao, Anal. Methods, 2021, 13, 862-873.

17 B. Li, D. Chen, J. Wang, Z. Yan, L. Jiang, D. Duan, J. He, Z. Luo, J. Zhang and F. Yuan, Sci. Rep., 2014, 4, $39-43$.

H. G. T. Ly, G. Fu, A. Kondinski, B. Bueken, D. De Vos and T. N. Parac-Vogt, J. Am. Chem. Soc., 2018, 140, 6325-6335.

H. G. T. Ly, G. Fu, F. de Azambuja, D. De Vos and T. N. Parac-Vogt, ACS Appl. Nano Mater., 2020, 3, 8931-8938.

20 A. Loosen, F. de Azambuja, S. Smolders, J. Moons, C. Simms, D. De Vos and T. N. Parac-Vogt, Chem. Sci., 2020, 11, 6662-6669.

21 L. Tsiatsiani and A. J. Heck, FEBS J, 2015, 282, 2612-2626.

22 D. L. Swaney, C. D. Wenger and J. J. Coon, J. Proteome Res., 2010, 9, 1323-1329.

23 N. E. Wezynfeld, T. Frączyk and W. Bal, Coord. Chem. Rev., 2016, 327-328, 166-187.

24 S. Mahesh, K. C. Tang and M. Raj, Molecules, 2018, 23, 1-43. 
G. Allen and R. Campbell, Int. J. Pept. Protein Res., 1996, 48, 265-273.

G. Allen, in Met Ions Biol Syst, CRC Press, Boca Raton, Florida, 38th edn., 2001, pp. 197-212.

F. de Azambuja, J. Moons and T. N. Parac-Vogt, Acc. Chem. Res., 2021, 54, 1673-1684.

K. Grant and M. Kassai, Curr. Org. Chem., 2006, 10, 1035-1049.

L. Vandebroek, E. De Zitter, H. G. T. Ly, D. Conić, T. Mihaylov, A. Sap, P. Proost, K. Pierloot, L. Van Meervelt and T. N. Parac-Vogt, Chem. Eur. J., 2018, 24, 10099-10108.

A. V Anyushin, A. Sap, T. Quanten, P. Proost and T. N. Parac-Vogt, Front. Chem., 2018, 6, 1-9.

J. Moons, F. Azambuja, J. Mihailovic, K. Kozma, K. Smiljanic, M. Amiri, T. Cirkovic Velickovic, M. Nyman and T. N. Parac-Vogt, Angew. Chemie Int. Ed., 2020, 59, 9094-9101.

M. H. Beyzavi, R. C. Klet, S. Tussupbayev, J. Borycz, N. A. Vermeulen, C. J. Cramer, J. F. Stoddart, J. T. Hupp and O. K. Farha, J. Am. Chem. Soc., 2014, 136, 15861-15864.

P. García-García and A. Corma, Isr. J. Chem., 2018, 58, 1062-1074.

M. Rimoldi, A. J. Howarth, M. R. Destefano, L. Lin, S. Goswami, P. Li, J. T. Hupp and O. K. Farha, ACS Catal., 2017, 7, 997-1014.

L. H. T. Nguyen, T. T. T. Nguyen, P. H. Tran, Y. Kawazoe, H. M. Le and T. L. H. Doan, J. Catal., 2019, 374, 110-117.

P. Li, R. C. Klet, S. Y. Moon, T. C. Wang, P. Deria, A. W. Peters, B. M. Klahr, H. J. Park, S. S. Al-Juaid, J. T. Hupp and O. K. Farha, Chem. Commun., 2015, 51, 10925-10928.

A. Radzicka and R. Wolfenden, J. Am. Chem. Soc., 1996, 118, 6105-6109.

D. Conic, K. Pierloot, T. N. Parac-Vogt and J. N. Harvey, Phys. Chem. Chem. Phys., 2020, 22, 25136-25145.

41 A. E. Counterman and D. E. Clemmer, J. Am. Chem. Soc., 1999, 121, 4031-4039. 